\title{
Energy Demands and Metabolic Equivalents (METS) in U-19 Basketball Refereeing During Official Games
}

\author{
Mohamed Ali Nabli ${ }^{1} 2^{*}$, Nidhal Ben Abdelkrim ${ }^{1}$, Carlo Castagna ${ }^{3}$, Imed Jabri ${ }^{4}$, Tahar Batikh ${ }^{4}$ and Karim Chamari $^{5}$ \\ ${ }^{1}$ Tunisian Research Laboratory Sports Performance Optimization, National Center of Medicine and Science in Sports (CNMSS), Tunis, Tunisia \\ ${ }^{2}$ Department of Biology, Faculty of Sciences of Bizerte, University of Carthage, Bizerte, Tunisia \\ ${ }^{3}$ School of Sport and Exercise Sciences, Faculty of Medicine and Surgery, University of Rome Tor Vergata, Rome, Italy \\ ${ }^{4}$ Higher School of Sciences and Techniques of Tunis, University of Tunis, Tunis, Tunisia \\ ${ }^{5}$ Athlete Health and Performance Research Centre, Qatar Orthopedic and Sports Medicine Hospital, Doha, Qatar
}

*Corresponding author: Mohamed Ali Nabli, Faculty of Sciences of Bizerte, University of Carthage, Tunisia, Tel: +974 6651377 / +216 55 947040; E-mail: nablimedali@live.fr

Received date: February 24, 2017; Accepted date: March 30, 2017; Published date: April 7, 2017

Copyright: (C) 2017 Nabli MA, et al. This is an open-access article distributed under the terms of the Creative Commons Attribution License, which permits unrestricted use, distribution and reproduction in any medium, provided the original author and source are credited.

\begin{tabular}{|l|} 
Abstract \\
The metabolic demands among $U-19$ basketball players were studied; however, physical and physiological \\
demands of $U-19$ basketball refereeing during competition are rather scant. The aim of this study was to analyze the \\
energy expenditure (EE) and the exercise intensity of basketball refereeing during official game and determined as \\
Metabolic Equivalent (METs). Sixteen elite level basketball referees were studied during U-19 basketball games \\
$(\mathrm{n}=8)$ for time motion analyses (TMA). The EE data were calculated, using specific equations, from the time spent by \\
the referee in each exercise-intensity zone. During game, referees spent a mean EE of $504.4 \pm 77.7 \mathrm{kcal}$. A \\
significant difference was observed between $113.5 \pm 18.2 \mathrm{kcal}$ in the 1 st quarter $(\mathrm{Q} 1)$ and $137 \pm 27.5 \mathrm{kcal}$ in the Q4 \\
$(\mathrm{p}=0.007)$. The averaged EE $\left(\sim 5 \mathrm{kcal}^{-1} \mathrm{~kg}^{-1} \mathrm{~h}^{-1}\right)$ corresponded to "moderate energy intensity" $(\sim 5 \mathrm{METs})$ with a large \\
contribution of the aerobic energy pathway. In all, during $88 \%$ of game the intensity was equal or lower than 3.8 \\
METs and in $12 \%$ it was higher than 9.8 METs which were considered as low to high-intensity thresholds, \\
respectively. The energetic profile studied in this research confirms that basketball refereeing may be regarded as a \\
clearly intermittent-activity mode with a predominantly aerobic metabolism solicitation. Referees officiating in $\mathrm{U}-19$ \\
basketball games should carry out a physical conditioning regime that prepares them for such an effort.
\end{tabular}

Keywords: Category; Recovery; Velocity; Oxygen consumption; Capacity

\section{Introduction}

Interest in quantifying energy expenditure (EE) in a variety of sports has increased dramatically in recent years. In fact, EE monitoring could provide information on energetic and metabolic demands during official competition. In basketball, the EE of players during a game is considered to range between 360 and $660 \mathrm{kcal} \mathrm{h}^{-1}$ [1]. In this context, the recommended energy intake is $4500-5000 \mathrm{kcal}$ for male basketball players [2]. Moreover, Salarkia et al. [3] reported that professional basketball player's energy requirement may equal to 3900 kcal day ${ }^{-1}$. But it's recognized that basketball player's energy requirement is not stable; $\mathrm{EE}$ could vary according to the changes in the frequency and intensity of training sessions, exercises and games [4].

The EE is the metabolic energy equivalent of the physical activity [5]. EE during activity could be measured to determine a diet that appropriately restores the athlete's EE during training and/or game [5]. Perfectly, EE should be quantified by measuring oxygen uptake $\left(\mathrm{VO}_{2}\right)$ in continuo during the game using indirect calorimetry [5]. However, this is not possible during official games, due to evident practical constraints. Even during training, the eventual use of metabolimeters is hardly considered for obvious reasons. Consequently, other variables, either tightly or basely related to EE and physical activity, have been considered and used. The heart-rate (HR), body-temperature [6], estimated $\mathrm{VO}_{2}$ [7], nutritional assessment and food intake [3], muscular creatine-phosphate and blood-lactate concentration [8], could be listed as a substitute variables. Osgnach et al. [9] relatively recently applied to the time motion analyses (TMA) values a computational approach to calculate EE during official soccer competitions. Nevertheless, compared to the amount of details collected on players, there is a deficiency of information describing the physical activity of referees during game [10].

There are many researches that tried to examine the EE on soccer referees during the match. Da Silva et al. [4] estimated the EE on Brazilian soccer referees during professional match. The soccer referee's estimated match energy demand varied from 3500 to 4000 $\mathrm{kcal} . d a y^{-1}$ [4]. Ardigò et al. [11] used the global positioning system (GPS) to quantify EE and physical activity variables during soccer refereeing. However, studies conducted on basketball referees are rather scant; the only available study [7] has analyzed the basketball referees' EE during the game by relating the $\mathrm{HR}$ response in the game to the laboratory-determined relationship between $\mathrm{HR}$ and $\mathrm{VO}_{2}$. Determination of $\mathrm{VO}_{2}$ requirement during the game permit the quantification of athlete's EE with thinkable accuracy [12]. Since, on average, $5 \mathrm{kcal}$ are resulted for 1 liter of consumed oxygen, it is possible to express EE during the game in kcal [4]. Also, the intensity of basketball referee's physical activity during games has been evaluated by the recording of HR [7].

Another means of estimating EE during the physical activity implies the statement in terms of Metabolic Equivalent (METs). For defining, a 
Page 2 of 6

MET represents the resting $\mathrm{VO}_{2}$ and is set as the $\mathrm{EE}\left(\mathrm{VO}_{2}\right)$ as $\mathrm{mlkg}^{-1} \mathrm{~min}^{-1}$ or Lmin ${ }^{-1}$ in rest conditions [13]. Consequently, the $\mathrm{EE}$ of a physical exercise can be defined in multiples of resting $\mathrm{VO}_{2}$, which simplifies the calculations of energy requirements during the effort. According to Wilmore et al. [14], all physical activity can be categorized by the intensity according to their requirements in oxygen. Therefore, through METs calculations, it is possible to categorize the intensity of different physical exercise [13]. Recent researches have showed that basketball refereeing is an activity model principally linked to the aerobic pathway $[15,16]$. However, it is also useful to convert this into an $\mathrm{EE}$ and the intensity of referee's physical activities performed during a game presented in METs to assist with conditioning and nutritional programs [4]. Therefore, the aim of the present study was to determine the EE and the intensity of the physical activity (expressed in METs) performed by U-19 basketball referees during a game.

\section{Materials and Methods}

\section{Participants}

This study included 16 male Tunisian referees (age: $35.7 \pm 9.11$ years, weight: $80.3 \pm 7.54 \mathrm{~kg}$, height: $1.80 \pm 0.07 \mathrm{~m}$ and body mass index: $24.7 \pm 1.51 \mathrm{~kg} \mathrm{~m}^{-2}$ ), 4 were classified as international referees and 12 as national referees with an average experience of $12 \pm 10$ years as basketball referees. They have been referees at senior level. The participants were observed during official games and each referee was measured on one occasion only. All referees had medical clearance for the physical activity. Referees were aware of the experimental protocol and risks and benefits without being aware of the aim of this study. They signed an informed written consent, before starting the investigation. The study protocol was in accordance with the guidelines planned by the World Medical Assembly Declaration of Helsinki and was approved by the University Ethics Committee [17].

\section{Study design}

This study was conducted during the play-off phase of the Tunisian U-19 basketball championship (April-June 2013, the season started in October 2012). In this study, a total of 8 official games of the play-off phase were considered. Games were performed on separate Sundays (one game per week) but all were held at the same hour (i.e., starting at 4:00 pm) and under close warm environmental conditions (i.e., $28-31^{\circ} \mathrm{C}$ ). The game-duration composed of four 10-min Q with a 15min interval at half-time (between 1st half: Q1+Q2 and 2nd half: Q3+Q4) and a 2-min break between the Q of each half (e.g. Q1 to Q2).

Referees' activities were assessed using video TMA. EE values were determined, using fixed equations; from the duration spent in each physical activity (see methods).

The data here provided are part of a main project that examined the game performance and fitness status of North African elite level basketball referees. The TMA data in this study was re-used from the original source [18] to analyze the referee's energy expenditure. Other sub-set data such as the physiological measures of the participants (i.e., heart rate and blood lactate concentration) were published elsewhere [18].

\section{Time-motion analysis (TMA)}

Game activities were monitored by means of a device using a technology similar to that reported by Ben Abdlekrim et al. [19]. Video recordings were collected using a fixed camcorder (Sony ${ }^{\circ}$, model Handycam CCD-TRV 128) tied to a tripod, installed $\sim 5 \mathrm{~m}$ far away from the sideline at half-court, at a height of $\sim 12 \mathrm{~m}$ (depending on the sport hall concerned) to permit full coverage of the court. The software adopted in this time-motion analysis (PC-foot ${ }^{\circledR} 4$ ) allowed the detecting of the referees' positions in each frame [20]. The court calibration was made by the same software using the dimensions that applied by the FIBA rules. The adopted algorithm was based on the subtraction of the background applying a statistic modelling, and comprises the following proceedings: initialization, motion-extraction and update of the model [19].

The video registration was analyzed frame by frame to a precision of 0.04 second. The video series could be visualized at any selected speed, allowing analysis of the motion. The following locomotive categories were used: standing/slow motion $\left(0-2.92 \mathrm{~km} \mathrm{~h}^{-1}\right)$, walking $(>2.92-7.34$ $\left.\mathrm{km} \mathrm{h}^{-1}\right)$, jogging $\left(>7.34-10.11 \mathrm{~km} \mathrm{~h}^{-1}\right)$, running $\left(>10.11-14.83 \mathrm{~km} \mathrm{~h}^{-1}\right)$ and sprinting $\left(>14.83 \mathrm{~km} \mathrm{~h}^{-1}\right)$, while the mean speed for each category was calculated after analysis of the video series. The time for the participant to pass known distances pre-markers in the court was used to calculate the speed for each motion activity. These categories were chosen in accordance with a previous study on soccer refereeing, in absence of any study on basketball [4]. Then these categories were classified in: (1) low-intensity activities; including standing/slow motion and walking (2) moderate to high intensity activities; including jogging, running and sprint.

A total-time refers to all of game-time comprising all playstoppages, but excluding breaks between $\mathrm{Q} /$ half times. The frequency and time spent in each motion category was digitally registered by the same experienced observer. These data were used to determine the referees' distance covered in each motion category. The total distance covered during each moment of the game was determined by adding the distances covered in each motion category. Reliability of TMA was studied by repeating the analysis of two games by the same experimenter one month apart. For each game the complete analysis of the 2 referees was performed and then reliability analysis was conducted. Game analysis and reliability assessments were performed by the same experimented investigator. According to Hopkins [21] the analyses of relative and absolute reliability of TMA have shown similar and good reliability (Table 1 ). These results of reliability were alike to that found in previous studies that involved soccer referees $[4,20,22]$ and basketball players [19].

\begin{tabular}{|l|l|l|}
\hline & ICC & \%SEM \\
\hline Walking & 0.99 & 0.005 \\
\hline Jogging & 0.99 & 0.52 \\
\hline Running & 0.74 & 0.58 \\
\hline Sprinting & 0.97 & 0.63 \\
\hline $\begin{array}{l}\text { ICC: Intra-class-coefficient; \%SEM: Standard error of measurement in } \\
\text { percentage }\end{array}$ & \\
\hline
\end{tabular}

Table 1: Relative and absolute reliability of time-motion measurement $(\mathrm{n}=4)$. 
Citation: Nabli MA, Abdelkrim NB, Castagna C, Jabri I, Batikh T, et al. (2017) Energy Demands and Metabolic Equivalents (METS) in U-19 Basketball Refereeing During Official Games. J Sports Med Doping Stud 7: 190. doi:10.4172/2161-0673.1000190

Page 3 of 6

\section{Energy expenditure (EE)}

EE values were determined from the time referees spent in each physical activity zone. The following equations were used to estimate the oxygen uptake $\left(\mathrm{VO}_{2}\right)$ during: (i) running and sprinting: $\mathrm{VO}_{2}=3.5$ $+\left(0.2 \times \mathrm{m} \mathrm{min}^{-1}\right)$, (ii) walking: $\mathrm{VO}_{2}=3.5+\left(0.1 \times \mathrm{m} \mathrm{min}^{-1}\right)$, both proposed by the American College of Sport Medicine [23]. $\mathrm{VO}_{2}$ during jogging was estimated by the equation proposed by Bubb et al. [24] $\mathrm{VO}_{2}=3.5+\left(\mathrm{km} \mathrm{h}^{-2} \times 0.394\right)$. EE during the time participants stayed standing-still was estimated multiplying the basal metabolism constant $3.5 \mathrm{ml} \mathrm{kg}^{-1} \mathrm{~min}^{-1}$ by the average body mass. Then, the value of estimated $\mathrm{VO}_{2}$ was transformed in kcal, multiplying it by 5 [14]

The quantification of referee's EE throughout the game in METs was possible by estimating the $\mathrm{VO}_{2}$ expended in each motion category [4]. For an adult, $1 \mathrm{MET}$ is equal to $\sim 3.5 \mathrm{ml}$ of oxygen uptake, for kilogram of body mass per minute $\left(1 \mathrm{MET}=3.5 \mathrm{mlkg}^{-1} \mathrm{~min}^{-1}\right)$. For example, a two MET activity request two times the metabolic EE of standing still. Therefore, the EE was estimated by dividing the estimated $\mathrm{VO}_{2}$ of each motion category by the constant 3.5 [4].

\section{Statistical analysis}

All data are presented as mean \pm standard deviation (SD). The normality's assumption was tested using the Shapiro-Wilk test for each variable before conducting any statistical parametric test. To compare in between $\mathrm{Q}$, analysis of variance (one-way ANOVA) was used. When necessary, the post-hoc Tukey's test was applied. To compare in between halves, an independent samples T-test was used. The relative and absolute reliability of the TMA were determined with Intra-classcoefficient (ICC) and using the standard error of measurement (SEM) [25], respectively. All statistical calculations were determined using SPSS 19 program for Windows (SPSS, Inc, Chicago, IL, USA). The significance level considered for all the statistical analysis was set at $\mathrm{p}<0.05$.

\section{Results}

Absolute and relative reliability calculations are reported in Table 1. TMA showed acceptable relative (ICC 0.70-0.99) and absolute reliability (\% SEM $0.005-0.63 \%$ ). Table 2 introduces the total average time spent in each physical activity zone during the game. Referees expend more of the time walking, and high-intensity activities (running and sprinting) amounted for $\sim 6$ minutes. Table 3 shows the mean whole distances covered in each motion category during game. The mean whole distance covered was $3907 \pm 848.5 \mathrm{~m}$, referees spent $81 \%$ of game time by walking, $7 \%$ by standing/slow motion, $6 \%$ by jogging, $4 \%$ by running, and $2 \%$ by sprinting.

\begin{tabular}{|l|l|lr|l|l|l|ll|}
\hline & Standing & Walking & $\begin{array}{l}\text { Joggin } \\
\mathbf{g}\end{array}$ & Running & Sprinting & \multicolumn{2}{|l|}{ Total } \\
\hline Game & $5.5 \pm 1.2$ & $\begin{array}{lll}6.5 .5 & \pm & 3.6 \\
2.1 & 0.2\end{array} \quad \pm$ & $4.2 \pm 0.3$ & $1.4 \pm 0.2$ & $\begin{array}{l}78.4 \\
4.2\end{array}$ & \pm \\
& & & & & & \\
\hline
\end{tabular}

The values represent the mean \pm SD of the duration of each motion action expressed in minutes

Table 2: Time in minutes spent in each motion activity $(\mathrm{n}=16)$.

Table 4 shows the results for the EE in each motion category. The entire game $\mathrm{EE}$ was $504.4 \pm 77.7 \mathrm{kcal}$ with significant differences observed between $113.5 \pm 18.2 \mathrm{kcal}$ in Q1 and $137 \pm 27.5 \mathrm{kcal}$ in Q4 $(\mathrm{p}=0.007)$. A means of $127.4 \pm 26.4 \mathrm{kcal}$ and $126.6 \pm 22.2 \mathrm{kcal}$, were observed as the referees' $\mathrm{EE}$ in Q2 and Q3, respectively. A nonsignificant $(\mathrm{p}=0.14)$ difference was observed between EE in the 1st and 2nd half $240.9 \pm 42.4$ and $263.6 \pm 42.9 \mathrm{kcal}$, respectively (Figures 1 and 2).

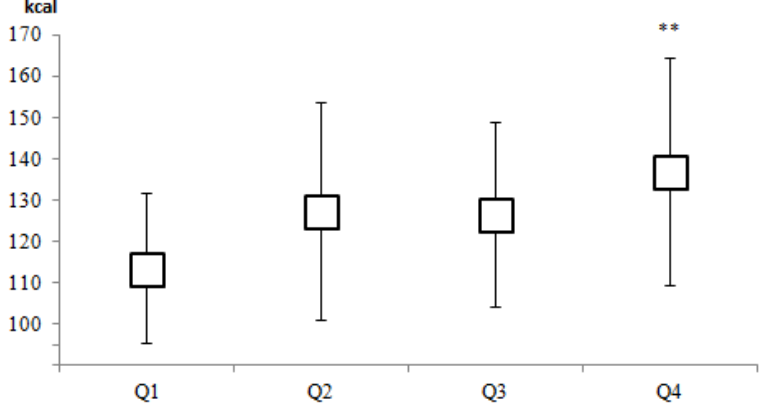

Figure 1: Summary of energy expenditure of basketball referees during quarters of official games. ${ }^{*}$ Significant difference between $\mathrm{Q} 1$ and $\mathrm{Q} 4$ ( $\mathrm{p}=0.007) ; \mathrm{Q}$, quarter.

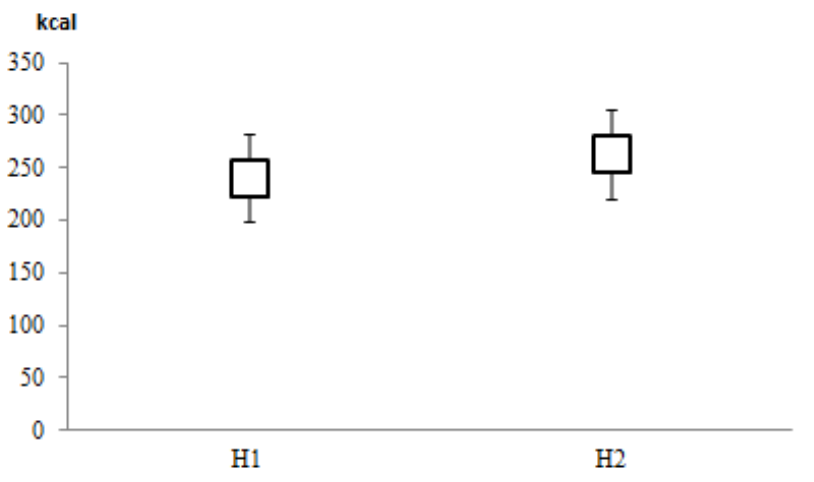

Figure 2: Summary of energy expenditure of basketball referees during halves of official games. $\mathrm{H}$ : half-time.

The mean EE was $\sim 5 \mathrm{kcal} \mathrm{kg}^{-1} \mathrm{~h}^{-1}$ that corresponded to $\sim 5$ METs. This was considered as moderate energy intensity. The referees spent $88 \%$ of the game time at walking and standing/slow motion as low intensity ( $3.8 \mathrm{METs})$ and $12 \%$ of the game time by jogging, running and sprinting as moderate to high intensity ( higher than 9.8 METs).

The large to bass-intensity exercise ratio may be expressed as $\sim 1: 12$. This reflects that an important-intensity action occur every $\sim 12$ lowintensity actions. The basketball referees expend 4357 seconds at bassintensity activities and 355 seconds at high-intensity.

\begin{tabular}{|l|l|l|l|l|}
\hline Walking & Jogging & Running & Sprinting & Total \\
\hline $2036 \pm 668$ & $447 \pm 139$ & $927 \pm 369$ & $486 \pm 186$ & $3907 \pm 848$ \\
\hline \multicolumn{4}{|l}{ The values represent the mean \pm SD. The distances are described in meters } \\
\hline
\end{tabular}

Table 3: Distance covered by referees during game-play $(n=16)$. 


\begin{tabular}{|l|l|l|l|l|l|l|}
\hline & $\begin{array}{l}\text { Speed } \\
\left(\mathbf{m ~ s}^{-1}\right)\end{array}$ & $\begin{array}{l}\text { Speed } \\
\left(\mathbf{m}_{\left.\mathbf{m i n}^{-1}\right)}\right.\end{array}$ & $\begin{array}{l}\text { Estimated } \\
\mathbf{V O}_{2} \mathbf{( m \mathbf { l }} \\
\left.\mathbf{k g}^{-1} \mathbf{m i n}^{-1}\right)\end{array}$ & $\begin{array}{l}\text { Estimated } \\
\mathbf{V O}_{2} \\
\left.\mathbf{m i n}^{-1}\right)\end{array}$ & $\begin{array}{l}\text { Time } \\
(\mathbf{m m}: \mathbf{s s})\end{array}$ & kcal \\
\hline Standing & & & 3.5 & 0.3 & 5.5 & 8.2 \\
\hline Walking & 1.6 & 97.5 & 13.3 & 1.1 & 63.5 & 338.9 \\
\hline Jogging & 2.5 & 147.9 & 34.5 & 2.8 & 3.6 & 42.7 \\
\hline Running & 3.2 & 189.8 & 41.5 & 3.4 & 4.2 & 72.2 \\
\hline Sprinting & 5.1 & 304.8 & 64.5 & 5.2 & 1.4 & 42.5 \\
\hline Total & & & & & 78.4 & 504.4 \\
\hline
\end{tabular}

$\mathrm{VO}_{2}$ : Oxygen uptake

Table 4: Calorific expenditure per motion action during game-play $(\mathrm{n}=16)$.

\section{Discussion}

This is the first research to investigate the energy expenditure (EE) of U-19 basketball referees during game after FIBA rule changes in May 2000. The latter changes consisted of reducing the attack duration from 30 to $24 \mathrm{~s}$ and the time permitted to get over the median-line from 10 to $8 \mathrm{~s}$, as well as, the game consisted of four 10-min quarters (Q) in place of two 20-min halves [19]. In the present study, the total game EE was $504.4 \pm 77.7 \mathrm{kcal}$ with a significant improvement in $\mathrm{EE}$ was showed in the 4 th quarter $(\mathrm{Q})$ compared to 1 st $\mathrm{Q}$. It was observed that during games referees performed large periods of bass-intensity exercise interspersed with short sequences of intense-exercise. In all, during $88 \%$ of game-time the intensity was lower than 3.8 METs, in $12 \%$ it was higher than 9.85 METs. Overall the results show that basketball refereeing may be defined as an intermittent exercise mode with permanent aerobic source [17].

During the game, referees covered a mean distance of $3906.94 \pm$ $848.5 \mathrm{~m}$. This value was similar to that observed in Borin 'study [16] during the qualifying phase of the competition in the Brazilian Basketball League (3026-4770 m), which officiated by the 3 referees system and involved the FIBA rules (game was consisted of $4 \times 10 \mathrm{~min}$ Q). But data were lower than those observed by Lončar et al. [26], who reported that basketball referees covered an average distance of $5921 \mathrm{~m}$ and $6773 \mathrm{~m}$ during a Slovenian Basketball League Play-off that officiated by 3 referees and 2 referees, respectively. These differences could be partially linked to different factors, such as; the competitive level of the analyzed games [4]. In fact, the distances covered by referees in a basketball game may be increased throughout competition phases [16].

However, the whole distance covered during a game is an insufficient indicator of the referee's physical requirements during a game, since standing/slow motion and walking account for $\sim 88 \%$ of total activity. In this context, the quantity of high-intensity exercise is a preferable informer of the demanding periods of the game and the evolution of fatigue [27].

An $81 \%$ of game time was performed by referees at a low intensity (i.e., walking). Also, they covered $52.1 \%$ of total distance by walking. The result in the present study was higher than that recorded previously [26] in Slovenian basketball referees during games officiated by 2 and 3 referees $(32.4 \%$ and $37.5 \%$ of their total distance, respectively). Differences in game-tactics and game-intensity between countries/teams are other reasons that should also be taken into consideration when comparing results $[28,29]$. The high percentage (81\%) of exercise at bass-speed (walking) estimated in this study could be the consequence of an inferior game-intensity of the regional tournament where referees officiated, and/or differences in game character between North African and Europeans players. In addition, players' tactical strategies could also affect the referee's high-intensity exercise and especially in Q4 as during the last moments of a game, teams (i.e., U-19 basketball championship) are probably to manage a more dominance of ball possession [19].

Running at high (sprinting) and moderate-intensity velocities (running) are infrequently executed by basketball referees. In this study, running at high intensity (sprinting) didn't arise for more than $2 \%$ of entire time (Table 2). This was lower than what previously reported [25] in Slovenian basketball referees during games officiated by 2 and 3 referees $(5.4 \%$ and $3.1 \%$ of their refereeing time, respectively).

In this research, a basketball referee's estimated EE during the game was $504.4 \pm 77.7 \mathrm{kcal}$. In basketball, studies performed so far on EE of referees are relatively scant. Rupcic et al. [7] have estimated the energy consumption per min for each game period; energy consumption values were determined by $\mathrm{HR}-\mathrm{VO}_{2}$ relationship in laboratory conditions to estimate the EE. However, it is recognized that the treadmill protocol for determining $\mathrm{HR}-\mathrm{VO}_{2}$ could overestimate the game $\mathrm{VO}_{2}$. For example, direct measurements of $\mathrm{VO}_{2}$ in football referees during a game registered a value of $\mathrm{EE}$ of $1195 \mathrm{kcal}$, and the value quantified by the recording of $\mathrm{HR}$ was $1565 \mathrm{kcal}$, in average [30]. The equations applied in this study to estimate the $\mathrm{VO}_{2}$ during physical activity used the average velocity for each category. Also, to estimate basketball referee's EE, TMA in this study did not take into account some referees' movements, such as acceleration, deceleration and changing of direction. However, Osgnach et al. [9] reported that an important metabolic load was imposed on players not only over the maximally intensive moments of the game (knowing as high running speed) but every time acceleration is high, even when reached velocity is low. Moreover, because of the acceleration and deceleration phases, the variability in EE for the same total distance is approximately $15 \%$. But, researches on accelerated (or decelerated) running are very limited because of the difficulty in using an energy approach in examining these types of exercises.

The results of the present study revealed a significant difference observed between $113.5 \pm 18.2 \mathrm{kcal}$ in Q1 and $137 \pm 27.5 \mathrm{kcal}$ in Q4 $(\mathrm{p}=0.007)$. The EE was significantly increased in the 2 nd half by $\sim 4.5 \%$, a fact that could reflect the increase in low (walking) and moderate (running) activities performed during this period. Indeed, in the 2 nd half, the distance covered in running was higher than the 1st half $(t=3.45, p=0.004)$, the referees spent significantly more time in walking and running than the 1 st half $(\mathrm{t}=2.75, \mathrm{p}=0.009$ and $\mathrm{t}=3.95, \mathrm{p}=0.0004$, respectively). This result may be reached by a good referee's physical fitness. Indeed, a good physical preparation could improve the referees' ability to keep up with play [31]. Also, referee's thermoregulation and rehydration processes could affect their EE during last period of competition. Indeed, referee's rehydration during the half-time interval may contribute to enhance their performance towards the end of the game [32]. However, Rupcic et al. [6] haven't reported a significant differences in the EE between the 1st and 2nd half-time (14 \pm 2 vs 13.7 $\pm 1.9 \mathrm{kcal} \mathrm{min}^{-1}$ ), as well as between particular quarters of basketball games. 
The intensity of the physical activity couldn't be determined, when the whole or partial EE of each motion enunciated in kcal. However, it is possible to create another way to express the $\mathrm{EE}$ and also of classifying the physical activity according to its intensity, using MET, when the estimated $\mathrm{VO}_{2}$ of each motion action was assumed. As mentioned above referees spent $81 \%$ of the game-time at walking, an exercise with an estimated $\mathrm{VO}_{2}$ of $13.25 \mathrm{ml} \mathrm{kg} \mathrm{min}^{-1}$, which equivalents to an EE of 3.8 METs. Also, $7 \%$ of the game-time was spent in standing/slow motion, with an estimated $\mathrm{VO}_{2}$ of $3.5 \mathrm{ml} \mathrm{kg}^{-1} \mathrm{~min}^{-1}$, and an EE of 1 MET. Consequently, during $88 \%$ of the game, they execute physical activities that could be categorized as low-intensity, aerobics $[13,33]$. The rest $12 \%$ of game-time is expended performing the physical activity using energy deriving from the aerobic and anaerobic pathways (jogging, running and sprinting). In jogging, the estimated $\mathrm{VO}_{2}$ was $34.5 \mathrm{ml} \mathrm{kg}-1 \mathrm{~min}^{-1}$, which corresponds to 9.9 METs, an effort expressed as intense [33]. During 6\% of the game time, referees performed running or sprinting motions, with an estimated $\mathrm{EE}$ of $41.5 \mathrm{VO}_{2} \mathrm{ml} \mathrm{kg}^{-1} \mathrm{~min}^{-1}$ (11.9 METs) and $64.5 \mathrm{VO}_{2} \mathrm{ml} \mathrm{kg}^{-1} \mathrm{~min}^{-1}$ (18.4 METs), respectively. Activities with energy consumption above 10 METs are classified as highly-intense [33]. Moreover, assessing energy intensity (i.e., MET) during games could provide information on referee's exercise load, with the related potential physiological adaptations. This study showed a specific ratio $(\sim 1: 12)$ between high and low intensity exercises in basketball referees, which reflect their specific intermittent activity during official games.

The EE of basketball referees may vary according to many elements, such as (i) the level of competition (senior vs U-19). For instance, the games of the final phases could be played with a higher physical and physiological stress than qualifying or semi-final phases [16], (ii) tactical strategies; the ways how teams play their offensive or defense systems in each period of the game, may impact the referees' demands/ responses. Indeed, during fast break action referees may require to perform a sprint to relocate themselves in strategical position with respect to player's action in the court, which impose a high EE, inversely to the zone offense playing style, where much less fast breaks are observed [19].

To get an objective measure of the average intensity of referee's physical activity during the game, the mediated average was used rather than the simple arithmetic average [4]. The amount of METs expenditure depends on the velocity, distributed in five categories in the present study. For instance, the part of METs spent in the condition "standing/slow motion" is far lower compared to sprinting. Besides, there is an important difference in the whole time of permanence in activities of high and low-velocity. Therefore, determination of the mediated average clearly decreases the possibility of obtaining biased outcomes by the highlighted reasons [4].

Based on the result of this research and using the classification adopted by Ainsworth et al. [13], it is feasible to identify the referee's physical activity as moderate energy intensity. This outcomes coincides with the values found in studies that estimated the intensity of referee's physical activity by means of HR $[15,16]$.

The METs method was established to unify intensities of physical activity and improve comparison different researches [13]. The METs data found in this study, allows comparing the intensity of a referee's physical activity with the outcomes obtained in referees or athletes from other sports. The latter referees and athletes were indeed studied more precisely and their energy requirements were better established.
Specific nutritional programs for basketball referees should be appointed founded on physical and metabolic requirements tried during official games $\left(\sim 5 \mathrm{kcal} \mathrm{kg}^{-1} \mathrm{~h}^{-1}\right)$. The dietary habits must be suitable to their quotidian physical activities. Indeed, referees should develop a diet that relatively restores the referee's EE during training and/or official game. Therefore, the training session should focus on intermittent aerobic-exercise of low and moderate intensity and frequent maximal-intensity (sprints) bouts with a $\sim 1: 12$ ratio of high intensity effort: recovery.

\section{Conclusion}

This study showed that during games from the Tunisian U-19 basketball championship referees covered on average, a distance of $3907 \pm 848.5 \mathrm{~m}$ with a mean energy EE of $504.4 \pm 77 \mathrm{kcal}$ per game. Averagely, this physical activity corresponds to moderate energyintensity ( $\sim$ METs) with large participation of the aerobic-energy pathway. However, during $88 \%$ of game-time the intensity was equal or lower than 3.8 METs (threshold of low intensity) and in 12\% higher than 9.8 METs (threshold of high intensity). The character of activity observed in this statement justify that basketball referees, like players assume intermittent nature exercise.

\section{References}

1. Nieman DC (1990) The immune response to prolonged cardiorespiratory exercise. Am J Sports Medicine 24: 98-103.

2. Rogozkin VA (1993) Principles of athlete's nutrition. In: Simpopoulos AP, Pavlou KN (eds). Nutrition and Fitness for Athletes. World Rev Nutr Diet Basel 71: 54-162.

3. Salarkia N, Kimiagar M, Aminpour A (2004) Food intake, body composition and endurance capacity of national basketball team players in I.R. of Iran. Medical Journal of the Islamic Republic of Iran 18: 73-77.

4. Da Silva AI, Fernandes LC, Fernandez R (2008) Energy expenditure and intensity of physical activity in soccer referees during match-play. J Sports Sci Med 7: 327-334.

5. Lamonte MJ, Ainsworth BE (2001) Quantifying energy expenditure and physical activity in the context of dose response. Med Sci Sports Exerc 33: 370-378.

6. Mohr M, Krustrup P, Bangsbo J (2005) Fatigue in soccer: A brief review. J Sports Sci 23: 593-599.

7. Rupčić T, Matković BR, Knjaz D, Nedić A, Popek S (2012) Differences in physiological load of the referees with consideration to the period of the basketball game. Sport Logi 8: 51-56.

8. Krustrup P, Mohr M, Steensberg A, Bencke J, Kaer M, et al. (2006) Muscle and blood metabolites during a soccer game: Implications for sprint performance. Med Sci Sports Exerc 38: 1165-1174.

9. Osgnach C, Poser S, Bernardini R, Rinaldo R, di Prampero PE (2010) Energy cost and metabolic power in elite soccer: A new match analysis approach. Med Sci Sports Exercise 42: 170-178.

10. Castagna C, Abt G, D'Ottavio S (2007) Physiological aspects of soccer refereeing performance and training. Sports Med 37: 625-646.

11. Ardigò LP, Padulo J, Zuliani A, Capelli C (2015) A low-cost method for estimating energy expenditure during soccer refereeing. J Sports Sci 9: 1-6.

12. Daniels J, Daniels N (1992) Running economy of elite male and elite female distance runners. Med Sci Sports Exerc 24: 483-489.

13. Ainsworth BE, Haskell WL, Leon AS, Jacobs D, Montoye HJ, et al. (1993) Compendium of physical activities: classification of energy costs of human physical activities. Med Sci Sports Exerc 25: 71-80.

14. Wilmore JH, Costill DL (2005) Physiology of sport and exercise (3rd edn). Human Kinetics Publishers, Idaho.

15. Matković A, Rupčić T, Knjaz D (2014) Physiological load of referee during basketball games. Kinesiology 2: 258-265. 
Citation: Nabli MA, Abdelkrim NB, Castagna C, Jabri I, Batikh T, et al. (2017) Energy Demands and Metabolic Equivalents (METS) in U-19

16. Borin JP, Daniel JF, Bonganha V, de Moraes AM, Cavaglieri CR, et al. (2103) The distances covered by basketball referees in a match increase throughout the competition phases, with no change in physiological demand. J Sports Med 4: 193-198.

17. World Medical Association (2001) Ethical principles for medical research involving human subjects. Bulletin of the World Health Organization 79: 373-374.

18. Nabli MA, Ben Abdelkrim N, Jabri I, Batikh T, Castagna C, et al. (2016) Fitness field tests correlate to game performance in U-19-category basketball referees. Int J Sports Physiol Perform 11: 1005-1011.

19. Ben Abdlekrim N, El Fazaa S, El Ati J (2007) Time-motion analysis and physiological data of elite under- 19 Basketball players during competition. Br J Sports Med 41: 69-75.

20. Battikh T, Jabri I, Annabi M (2006) Automatic control of the positions and trajectories of football actors in order to measure their physical and tactical performance. The 10th World multi-conference on systemics, cybernetics and informatics. Orlando, FL, USA. pp: 16-19.

21. Hopkins WG (2000) Measures of reliability in sports medicine and science. Sports Med 30: 1-15.

22. Krustrup P, Bangsbo J (2001) Physiological demands of top-class soccer refereeing in relation to physical capacity: Effect of intense intermittent exercise training. J Sports Sci 19: 881-891.

23. American College of Sport Medicine (1998) Guidelines for graded exercise testing and exercise prescription (2nd edn). Lippincott Williams \& Wilkins, Philadelphia (PA).
24. Bubb WJ, Martin AD, Howley ET (1985) Predicting oxygen uptake during level walking at speed of $80-130 \mathrm{~m} / \mathrm{min}$. J Cardiopulm Rehabil 5: 462-465.

25. Hopkins WG (2007) Understanding statistics by using spreadsheets to generate and analyze samples. Sport Sci 11:23-36.

26. Lončar M, Dežman B, Licen S (2004) Tracking two and three officials with a computer. FIBA Assist Magazine 20: 40-43.

27. Bangsbo J, Lindquist F (1992) Comparison of various exercise tests with endurance performance during soccer in professional players. Int J Sports Med 13: 125-133.

28. Rebelo A, Silva S, Pereira N, Soares J (2002) Physical activity of soccer referees uring the match. Revista Portuguesa de Ciências do Desporto 2: 24-30.

29. Helsen W, Bultynck JB (2004) Physical and perceptual-cognitive demands of top-class refereeing in association football. J Sports Sci 22: 179-89.

30. Shephard R (1992) The energy needs of the soccer player. Clin J Sport Med 2: 62-70.

31. Weston M, Castagna C, Impellizzeri FM, Bizzini M, Williams AM, et al. (2012) Science and medicine applied to soccer refereeing an update. Sports Med 42: 615-631.

32. Reilly T, Gregson W (2006) Special populations: the referee and assistant referee. J Sports Sci 24: 795-801.

33. Durnin GB, Passmore R (1967) Energy, work and leisure. Heinmann, London. 